\title{
A Modularized Program For A Fully Automated Image Acquisition
}

\author{
Steffen Meyer, Dieter Typke, Jürgen M. Plitzko and Wolfgang Baumeister \\ Max-Planck-Institute for Biochemistry, Department of Molecular Structural Biology, Martinsried, \\ Germany
}

Cryo-electron microscopy is the main method used for single particle analysis in structural biology research. However, the quality of one electron micrograph recorded under low-dose and cryo conditions depends essentially on the signal-to-noise ratio (SNR) while the quantity of images (of the same macromolecular structure) determines the achievable resolution of a subsequent image analysis. Therefore, we have to collect as many images as we can get [1] to obtain structures to very high resolution down to $4.5 \AA$ [2]. The limiting factor in collecting manually a large number of electron micrographs is the time a microscopist needs to setup the microscope, localize and identify the particles and finally acquire the images. With the automation of this process we cannot only substantially reduce the time required to get a sufficient number of images but also increase the statistical significance of the collected data volume.

We have used Quantifoils [3] to develop an automated system for collecting electron micrographs from ice embedded specimens. Quantifoils are ideal for the automation, because the copper grids are coated with a thin carbon film and include holes with a fixed size and a given geometry. The program was implemented and tested with a Philips CM120 BioFilter and a Philips CM300 FEG transmission electron microscope. Both microscopes are equipped with a Gatan imaging filter (GIF), a Gatan CCD Camera and we used the script programming language under the commercially available Digital Micrograph software.

In an initialization step the program scans the orientation of the holey-grid and centers a starting hole. At first the microscope and camera parameters have to be specified for the different steps in the automation cycle. This includes e.g. the magnification and illumination conditions, defocus setting, image size and the exposure time. There are basically three different settings for 'tracking', 'focus', and 'exposure'. The automatic scanning process ('tracking') starts with centering the next successive hole (Figure 1A) while simultaneously determining the 'quality' of the hole. If the centered hole meets some given criteria (e.g. sample/ice thickness) the program continues with adjusting the focus ('focus') [4], astigmatism and the acquisition of the final image ('exposure'). 'Tracking' to the next hole is done in a $\mathrm{z}$-shaped loop until an entire grid cell is thoroughly processed.

We have chosen a modular program structure to simplify further modifications and developments to the main scanning program. The main routine performs the 'tracking' part while external modules are used for 'quality' determination, 'focus' and 'exposure' (Figure 1A). Currently we have implemented 
modules for 'quality' (ice thicknes determination), 'focus' (focus and astigmatism correction) and 'exposure' (acquire one (Figure 1B), four or nine images per hole (Figure 1C)).

Utilizing the Gatan imaging filter we can determine the ice thickness very accurately (log-ratio technique) which we use as one quality criteria of the investigated hole [5]. Additionally, we have extended our focus routine to prevent irregularities, introduced by hysteresis effects of the objective lens. During the tracking procedure, we focus by readjusting the eucentrical height of the specimen rather than changing the objective lens current [6]. Furthermore, focus measuring can be done not only inside the hole but also in its surroundings, to minimize possible radiation damage. In the current state the program runs flawless for more than 10 hours. Assuming the presence of 20-30 particles in each image, we can automatically collect over 25.000 particle projections during this time where only the liquid nitrogen dewar has to be refilled periodically.

We will present results on various specimens and we will compare our findings in respect to the already established Leginon routines [7].

\section{References}

1. Henderson, R., Quart. Rev. Biophys. 28 (1995) 171-193.

2. Van Heel, M. et al., Quart. Rev. Biophys. 33 (2000) 307-369.

3. Ermantraut., E., Wohlfart, K. and Tichelaar, W., Ultramicroscopy 74 (1998) 75-81.

4. Koster, A.J. and de Ruijter, W.J., Ultramicroscopy 40 (1992) 89-107.

5. Malis, T., Cheng, S.C. and Egerton, R.F., J. Electr. Microsc. Tech. 8, (1988) 193-200.

6. Dierksen, K., Typke, D. and Hegerl, R., Ultramicroscopy 49 (1993) 109-120.

7. Carragher, B., Kisseberth, N., Kriegman, D., Milligan, R., Potter, C.S., Pulokas, J. and Reilein, A., J. Struct. Biol. 132 (2000) 33-45.
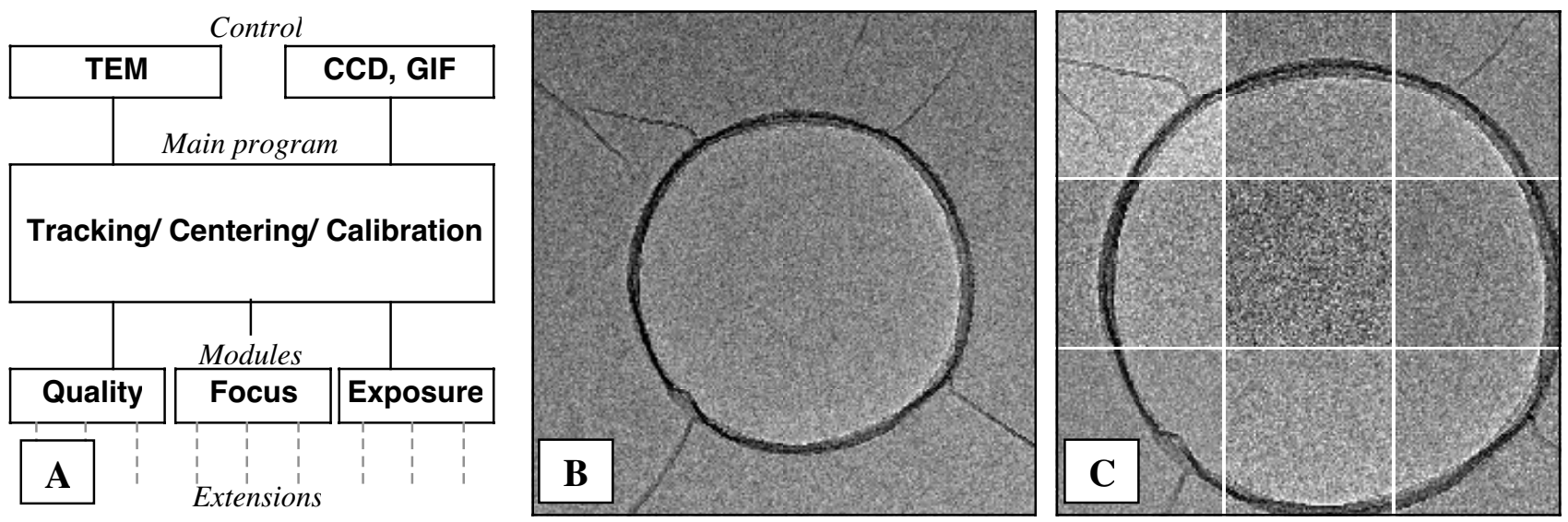

Figure 1: (A) Schematic of the modularized automation program. (B) 'Exposure' module for one and $(\mathbf{C})$ for nine images from one Quantifoil hole $(\varnothing \approx 2 \mu \mathrm{m})$. 